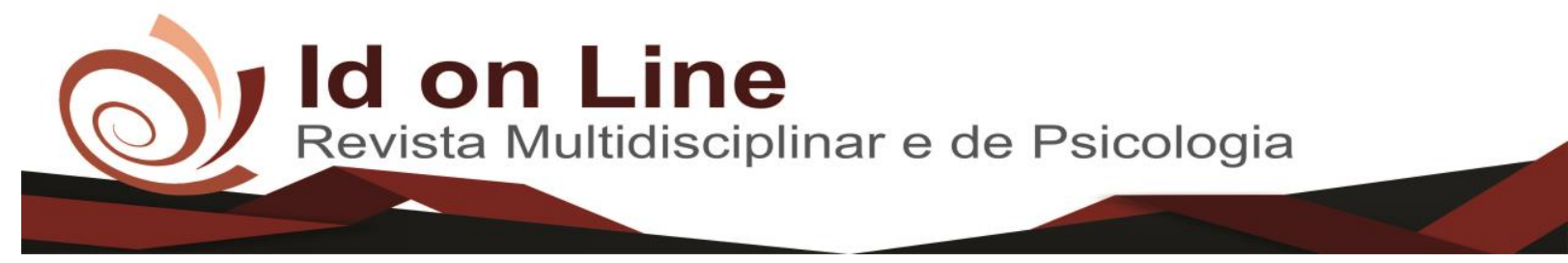

Artigo

\title{
A Formação do Enfermeiro para a Realização da Educação Continuada
}

\author{
Vinareis Gomes Da Silva ${ }^{1}$; Aldrina da Silva Confessor Cândido ${ }^{2}$
}

\begin{abstract}
Resumo: A educação em saúde está presente nos diversos locais e áreas de atuação do enfermeiro, desde a Atenção Primária em Saúde aos setores especializados. Objetivou-se com este trabalho conhecer como os enfermeiros constroem suas posturas pedagógicas para realização de atividades de educação continuada em um hospital privado do município de Vitória da Conquista - Bahia. Trata-se de um estudo qualitativo de natureza exploratória. A presente pesquisa foi realizada em um Hospital Privado, de médio porte, do interior da Bahia, no período de fevereiro a março de 2018. Os participantes da pesquisa foram cinco profissionais de nível superior (enfermeiros), das diversas clínicas e setores do hospital. O estudo mostrou que os profissionais, apesar da ausência de formação pedagógica para tal, se dizem aptos para realizar educação continuada no local de trabalho. Alguns profissionais ainda fazem Educação Continuada de forma tradicional, baseada apenas no repasse de conhecimentos, o que reforça a necessidade de quebrar-se barreiras quanto ao uso das metodologias ativas como método de ensino, desde a graduação. Foram citados vários fatores relacionados aos desafios e possibilidades para realização da Educação Continuada. Considera-se ao final a necessidade de fomentar as discussões sobre práticas pedagógicas na saúde para formação contínua de trabalhadores em saúde.
\end{abstract}

Palavras-Chave: Educação Continuada; Enfermeiros; Unidade hospitalar.

\section{Nursing Education for Continuing Education}

\begin{abstract}
Health Education is present in an amount of location and areas of practice of nurses, from e Primary Health Care, as well in specialized sectors. This study aimed to know how professionals to develop pedagogical postures for conductings continuing education practices in a private hospital in the city of Vitoria da ConquistaBA. This is a qualitative study that has exploratory nature. This research was conducted in a medium business private Hospital, in the interior of Bahia, from february to March, 2018. Survey respondents were five top-level professionals (nurses), from diverse clinics and hospital sectors. The study showed that the professional, they feel able to accomplish education continued in the workplace. Some professional develop continue education based on traditional model, only transferring knowledge which reinforces the need to break barriers regarding the use of active methodology as a method of teaching. Highlights the need for development of new research that covers the same topic, however that has greater scope in other fields of practice of nursing, such as Primary Health Care or specialized attention.
\end{abstract}

Keywords: Continuing Education; Nurses; Hospital Unit.

\footnotetext{
${ }^{1}$ Graduanda de Enfermagem da Faculdade Independente do Nordeste. Contato: nara_gomez@ $@$ hotmail.com;

2 Docente do curso de Enfermagem da Faculdade Independente do Nordeste, Doutoranda em Humanidades e Arte com ênfase em Ciências da Educação. Contato: aldrina@ fainor.com.br.
} 


\section{Introdução}

A educação em saúde está presente nos diversos locais e áreas de atuação do enfermeiro, desde a Atenção Primária em Saúde, até os setores especializados, tendo como foco a educação em saúde da população. Compreende ainda, os processos de formação profissional, que envolve a formação inicial, acadêmica, a formação continuada e/ou permanente e a formação em serviço.

No que tange à educação em saúde para a população, esta é entendida como um processo que auxilia os indivíduos a adquirir e compreender atitudes e comportamentos relacionados ao cuidado em saúde, que devem ser incluídos no cotidiano de vida e que tenham objetivo de melhorar a qualidade de vida e saúde do paciente (ARRUDA, MOREIRA e ARAGÃO, 2014).

Em relação aos processos de educação em saúde voltados para profissionais de saúde, estes são ofertados por meio de Educação Continuada e visam auxiliar e qualificar o processo de trabalho dos profissionais ou oferecer conhecimentos acerca de determinada área (FLORES, OLIVEIRA e ZOCCHE, 2016).

A educação no trabalho, no contexto da saúde é uma importante ferramenta para construção de mudanças na sociedade, uma vez que é capaz de despertar novos modos e visões de produção de saúde, com intuito de prestar atendimento de qualidade e que provoque maior satisfação nos usuários. Nesta linha, a Educação Continuada para profissionais tem sido primordial para que os mesmos construam e aprimorem suas competências e habilidades (SALUM e PRADO, 2014).

Assim, acredita-se que a Educação Continuada é fundamental para constituir em formas de garantir a manutenção da competência da equipe de enfermagem em relação à assistência. De acordo com as diretrizes curriculares para a formação dos profissionais de saúde, tem-se, especialmente para a graduação de enfermagem, a educação permanente como um dos requisitos básicos para a prática profissional (JESUS et al., 2011).

A motivação que levou à escolha do tema foi o intuito de contribuir com o conhecimento de uma questão de grande relevância para os profissionais de enfermagem: construção de posturas pedagógicas para realização de Educação Continuada. Do ponto de vista acadêmico, o assunto é de amplo interesse para os profissionais que realizarão futuramente este tipo de atividade. Não se trata apenas de interesse pessoal e acadêmico, haja vista a importância da Educação Continuada em saúde para a qualidade do cuidado. Portanto, têm-se a seguinte 
problematização a ser respondida por este trabalho: como os enfermeiros constroem suas posturas pedagógicas para realização de atividades de educação continuada? Acredita-se que a educação continuada tem potencial transformador no cotidiano de trabalho dos profissionais, no entanto, verifica-se que apesar da importância desta, a prática de educação continuada ainda é realizada de forma ineficiente.

Desta forma, objetiva-se conhecer como os enfermeiros constroem suas posturas pedagógicas para realização de atividades de educação continuada em um hospital privado do município de Vitória da Conquista - BA.

\section{Metodologia}

Trata-se de um estudo qualitativo, que possui natureza exploratória. As pesquisas qualitativas "buscam explicar o porquê das coisas, exprimindo o que convém ser feito, mas não quantificam os valores e as trocas simbólicas nem se submetem à prova de fatos, pois os dados analisados são não-métricos (suscitados e de interação) e se valem de diferentes abordagens" (GERHARDT e SILVEIRA, 2009, p.31). Já as pesquisas exploratórias têm a finalidade de proporcionar, desenvolver, esclarecer conceitos e ideias. Ressalta-se que, de acordo Gil (2010) o objetivo primordial é direcionar o pesquisador para um olhar global do tipo de determinado fato ou fenômeno.

A presente pesquisa foi realizada em um Hospital Privado, de médio porte, do interior da Bahia, no período de fevereiro a março de 2018. O Hospital possui diversas especialidades como cardiologia, neurologia, angiologia, ortopedia, dentre diversas outras e realiza cirurgias de médio porte. Também dispõe de UTI geral e cardiológica. Os participantes da pesquisa foram cinco profissionais de nível superior (enfermeiros), das diversas clínicas e setores do hospital. Foram adotados os seguintes critérios de inclusão: enfermeiros que trabalhavam há pelo menos três meses na unidade, que realizavam atividades de Educação Continuada para a equipe, que aceitaram participar voluntariamente da pesquisa e assinaram o Termo de Consentimento Livre e Esclarecido - TCLE. Foram excluídos aqueles que não aceitaram participar da pesquisa, não realizavam atividades de Educação Continuada ou não assinaram o TCLE.

Foi utilizado um roteiro semiestruturado, com questões abertas, elaboradas pelas próprias autoras do estudo. Os dados subjetivos foram analisados à luz da Análise de Conteúdo 
de Bardin e alocados em categorias de acordo a proximidade das respostas. Foi utilizado o Software Microsoft Office Word em versão 2010 para transcrição e organização dos dados.

O projeto foi encaminhado à direção do Hospital para apreciação e após resposta positiva o mesmo foi encaminhado ao Comitê de Ética em Pesquisa (CEP) da Faculdade Independente do Nordeste (FAINOR) tendo se iniciado a coleta de dados apenas após parecer favorável sob o $n^{\circ}$ 2.531.054. A pesquisa obedeceu todos preceitos da Resolução 466 de 2012 e Resolução 510 de 2016, sendo respeitados a dignidade e autonomia dos participantes da pesquisa, reconhecendo sua vulnerabilidade, assegurando sua vontade sob forma de manifestação expressa, livre e esclarecida, de contribuir e permanecer ou não na pesquisa.

Os participantes da pesquisa foram devidamente orientados quanto aos objetivos da pesquisa, ficando os mesmos livres para participarem ou não. Os profissionais que aceitaram participar assinaram o TCLE, redigido de maneira simples em linguagem clara e acessível aos participantes, em duas vias ficando uma cópia com o sujeito e a outra com a pesquisadora.

\section{Resultados e Discussões}

A população do estudo foi composta por cinco profissionais de enfermagem de nível superior, todas do sexo feminino, com faixa etária entre 30 e 36 anos de idade. O tempo de formação variou entre 02 e 14 anos de formada. Quanto ao tempo de trabalho na instituição também variou entre 02 e 14 anos.

Após análise das entrevistas, puderam-se criar algumas categorias de discussão: 01. Desenvolvendo educação continuada no ambiente de trabalho; 02. Papel da graduação no processo de desenvolvimento de habilidades para prática de educação continuada; 03. Desafios e potencialidades para desenvolvimento de educação continuada.

\section{Desenvolvendo Educação Continuada no Local de Trabalho}

Dentre as competências e habilidades gerais requeridas dos profissionais de Enfermagem encontra-se a educação permanente que se refere à necessidade do enfermeiro de comprometer-se com a formação, não só de futuras gerações, mas também dos profissionais nos serviços (BRASIL, 2001). Nesse contexto, identifica-se a Educação Continuada como 
sendo uma responsabilidade do profissional de enfermagem no intuito de por em prática a formação contínua nos serviços de saúde.

A análise das informações coletadas nos permitiu identificar que todas as profissionais que participaram da pesquisa realizavam Educação Continuada com uma frequência regular, conforme relatos a seguir:

"Faço educação continuada uma vez ao mês" (Enf. 01).

"Realizo a educação continuada uma vez por mês, dentro da carga horária (...)" (Enf. 04).

De acordo Silva e Seiffert apud Souza e Lima (2015), a Educação Continuada é algo dinâmico, são práticas usuais que objetivam mudanças no modelo hegemônico de saúde, bem como empodera o sujeito profissional para aquisição e busca de conhecimentos que reflitam diretamente na assistência prestada ao usuário.

Sabe-se que, para que a Educação Continuada cumpra seu papel formador, é essencial que seja realizada por profissionais competentes para tal. Buscou-se então, verificar, a partir da visão das enfermeiras entrevistadas, se estas se sentiam aptas ou não a realiza-la, constatandose que as profissionais se sentem aptas para realizar a Educação Continuada, como verificado nas falas abaixo:

"Sim, devido o tempo de experiência na área, proveniente também dos cursos realizados nesta área” (Enf. 03).

“Sim. Devido os cursos de atualidades e a busca continua do conhecimento" (Enf. 01).

Quanto ao planejamento e métodos utilizados para realização da Educação Continuada, observou-se que, o motivo da escolha do tema considerava as necessidades da equipe; que os recursos pedagógicos escolhidos valorizavam a exposição e que o profissional assumia uma postura, na sua maioria, centralizadora, pois, ele era quem planejava e executava a Educação Continuada, como mostrado nas falas abaixo:

\footnotetext{
"Desenvolvo a educação continuada baseado nas necessidades da equipe, realizo em conjunto definição de tema e, posteriormente, elaboro a aula com apresentação em data show e aula expositiva e dialogada" (Enf. 05).

“A elaboração do tema para a educação continuada é de acordo com a necessidade do trabalhador. Elaboro o plano de capacitação, o método, ministro, avalio resultados esperados, escolho público alvo e avalio o processo" (Enf. 02).
} 
A rotina das ações do enfermeiro é permeada por práticas pedagógicas, sejam elas formais ou informais, para tal ele deve compreender e ter olhar amplo sobre todas as variáveis entrelaçadas no processo de educação, o que é ainda hoje um desafio a ser superado, haja vista a presença marcante da formação do profissionais a partir da perspectiva tradicional, refletindo diretamente na forma de desenvolver Educação Continuada (BAPTISTA e CUNHA, 2009), como observado em algumas falas de profissionais que utilizam aulas expositivas e utilização de data show.

\footnotetext{
"Para a educação continuada realizo aulas expositivas em power point, descrevendo o tema abordado, com discussões de estudo de caso e situações relevantes" (Enf. 03).

“(...) buscando estabelecer relações, conceitos entre teoria, prática e realidade levando em conta a necessidade do setor e os objetivos supostos, os conhecimentos prévios do grupo e as limitações de tempo e recursos" (Enf. 04).
}

Observou-se, portanto, que a metodologia utilizada era predominantemente baseada em uma concepção tradicional, centrada no enfermeiro, o que refletia uma reprodução da prática pedagógica vivenciada pelas enfermeiras na maior parte da vida, fruto da ausência de uma formação pedagógica, que poderia contribuir para a execução da sua atribuição profissional relacionada à educação permanente.

O uso de recursos como aula expositiva e Datashow reforça a concepção tradicional implícita na atuação do enfermeiro na Educação Continuada, enquanto que, considerar as necessidades do grupo, os conhecimentos prévios e realizar discussões de casos clínicos, aproxima o enfermeiro do uso de uma metodologia mais participativa e menos tradicional.

Como superação desse modelo tradicional, na busca do desenvolvimento de posturas pedagógicas para desenvolvimento de Educação Continuada, é fundamental, que os profissionais de enfermagem busquem o conhecimento, busquem sanar dúvidas através de construção de conhecimentos e saberes crítico e reflexivos, que favorecerão os mesmos em momentos de dúvidas, conflito de valores, etc. (OLIVEIRA, 2013).

Desta forma, é extremamente importante que os profissionais ao desenvolverem Educação Continuada, promovam estratégias que estimulem a busca contínua do conhecimento e estimulem a criatividade por parte dos profissionais dos serviços que participarão da educação continuada. Do mesmo modo, é extremamente relevante a escolha do tema baseado nas necessidades da equipe, mas também ressalta-se, a necessidade de envolver a equipe neste planejamento e execução da Educação Continuada, não apenas permitindo a escolha de temas, mas mudando a metodologia de trabalho, priorizando uma Educação Continuada que valorize 
a troca de experiências e a reflexão sobre a prática profissional, para que os profissionais se sintam participantes do processo e não meros expectadores.

\section{Papel da Graduação no Processo de Desenvolvimento de Habilidades para prática de Educação Continuada}

As falas das enfermeiras entrevistadas foram analisadas no intuito de identificar as contribuições da graduação no desenvolvimento de competências e habilidade para atuar na Educação Permanente e, consequentemente, na Educação Continuada. Apesar de não citarem a existência de capacitações e/ou preparos pedagógicos para realizarem educação continuada, todos concordaram que a graduação colaborou para aquisição dessa habilidade. No entanto, uma das falas se destacou por apontar uma responsabilidade pessoal em desenvolver tal habilidade para exercer a Educação Continuada, conforme narrativas a seguir:

\footnotetext{
"A graduação colaborou, pois trouxe a prática da habilidade bem como incentivou o processo de aprendizado, pesquisa, treinamento, promovendo momentos para isso e orientações acerca da temática" (Enf. 02).

"Esse processo depende muito, na minha opinião, do interesse do aluno em querer desenvolver habilidade para discursar. Estar sempre apresentando trabalhos, não ter "medo" ou receio em disseminar informações e ir se aperfeiçoando. No meu caso a graduação colaborou e muito no meu processo" (Enf. 03).
}

Ainda se observou que, as enfermeiras reconheciam a importância da formação pedagógica para a Educação Continuada, no entanto, a formação destas profissionais parece ter ocorrido de maneira pouco sistematizada, baseada no incentivo, no oferecimento de dicas e informações e no interesse pessoal em desenvolver a habilidade para atuar na Educação Continuada, como vemos nas falas a seguir:

\footnotetext{
"Favorece o desenvolvimento profissional, proporciona uma visão global de crescimento, compartilhamento de saberes, desenvolve autoconhecimento para lidar com dificuldades" (Enf. 01).

"Durante a graduação foi demonstrado tanto teoricamente como na prática a importância e os resultados alcançados com a elaboração acertiva da educação continuada. Foi de grande ajuda o acervo de dicas e informações repassadas durante a graduação, pois foi a base para a minha prática no trabalho" (Enf. 05).
}

Alguns estudos têm demonstrado que a formação dos profissionais de saúde, pautada na perspectiva da realização de Educação Continuada, é algo já presente na sociedade brasileira há algum tempo e existem políticas que a trazem como proposta estratégica capaz de contribuir 
para mudanças e quebras de paradigmas, de práticas pedagógicas ainda pautadas em metodologias que "engessam" o conhecimento (JESUS et al., 2011).

Nesta perspectiva, e baseado nas Diretrizes Curriculares Nacionais para o Curso de Graduação em Enfermagem (DCN/ ENF), especificamente na Resolução CNE/CES No 03 de 7/11/2001, os cursos de graduação devem articular educação e saúde, na perspectiva de formação de um profissional crítico e educador. Sabe-se que, infelizmente, alguns cursos de bacharelado, que utilizam metodologia tradicional de ensino, inevitavelmente ainda formam profissionais tecnicistas.

De acordo Ferreira Júnior et al. (2010), na área da enfermagem, o curso de bacharelado não oferece subsídios de formação com intuito educativo, ao contrário do curso de Licenciatura em enfermagem, que tem como principal característica, a formação do profissional, no enfoque de ações do enfermeiro nas atividades de ensino. O autor cita o exemplo do curso de Licenciatura em enfermagem ofertado na Universidade Federal do Rio Grande do Sul e como sua estruturação se dá em oito blocos distintos, que abordam a educação, o ensino como agente transformador.

Neste sentido, é extremamente relevante que os cursos de graduação busquem a quebra de paradigma de modelos de ensino tradicionais e superem o olhar fragmentado durante o processo de formação dos profissionais. As instituições de ensino e os próprios discentes devem explicitar compromisso e responsabilidade com alterações, quebrando a lógica centrada no professor e reafirmando a capacidade de valorizar a potencialidade dos indivíduos no processo de construção de saberes (BRASIL, 2004; ACILOI, 2015).

Só assim, através destas mudanças de paradigmas, as instituições de ensino e os profissionais, no âmbito de desenvolverem habilidades pedagógicas para Educação Continuada, poderão ater-se da condição de renovarem a si próprios (BRASIL, 2004).

\section{Desafios e Potencialidades para Desenvolvimento de Educação Continuada}

As enfermeiras entrevistadas foram questionadas quanto aos desafios e potencialidades da equipe para desenvolvimento de Educação Continuada. Quanto aos desafios enfrentados, foram citados aqueles referentes à aspectos institucionais (recursos materiais, humanos, acúmulo de trabalho, pouco incentivo), seguido dos desafios referentes aos aspectos pessoais 
(falta de motivação e falta de integração entre as equipes) e por último os desafios referentes a aspectos didáticos.

"Falta de recursos materiais e humanos e didáticos, a falta de motivação por parte dos profissionais de enfermagem acumulo de trabalho, falta de integração entre as equipes como também grande demanda do serviço" (Enf. 04).

"Pouco incentivo das empresas com os seus colaboradores (...) mantendo uma visão arcaica de não impulsionar o crescimento profissional do colaborador" (Enf. 01).

De acordo com Gubert e Prado (2011), como um dos desafios para realização de Educação Continuada, para além de recursos humanos e materiais, verifica-se influência da formação dos profissionais de forma fragmentada e disciplinar na sua ação, o que exige cada vez mais interdisciplinaridade entre os profissionais. A dificuldade da interdisciplinaridade é verificada uma vez que a formação educacional fragmentada gera "desconfortos" e despreparo para realizar a Educação Continuada.

Quanto as potencialidades para a realização da Educação Continuada, as enfermeiras citaram como fatores facilitadores do processo, em primeiro lugar, os referentes aos aspectos institucionais, tais como: carga horária, disponibilidade de local e data, tempo destinado ao preparo da Educação Continuada, disponibilização de materiais de simulação, como relatado a seguir:

"Carga horaria para reunir toda equipe necessária, disponibilidade de local data e horário, tempo para preparar material que aborde o tema escolhido, material expositivo para simular determinada situação" (Enf. 03).

"Público interessado, atualização positiva, bons meios de execução, bons resultados" (Enf. 02).

Ainda como potencialidades, em segundo lugar, foram citados fatores referentes aos aspectos pessoais, como: a autonomia para definir com a equipe as necessidades reais, o interesse, envolvimento e participação da equipe, como confirmam as falas a seguir:

\footnotetext{
"Envolver toda equipe de modo que a educação seja algo diferente do que se é rotina, trazer o colaborador para participação ativa de maneira que ele entenda que o envolvimento no processo é fundamental para o processo de aprendizagem e troca de saberes" (Enf. 05)

“Autonomia para avaliar a real necessidade da equipe" (Enf. 05).
}

De acordo Amaro et al. (2018), as potencialidades da educação permanente pautam-se em três pilares: profissional, usuário e serviço. O autor também cita que através da Educação 
Continuada é possível realizar transformações de práticas no cotidiano de trabalho. É possível verificar que os profissionais da presente pesquisa, têm a mesma visão e compreendem a necessidade de utilizar estratégias pedagógicas mais envolventes e que priorizem a participação colaborativa de toda a equipe.

\section{Considerações Finais}

Pode-se concluir, que o trabalho conseguiu atingir o objetivo proposto inicialmente, que foi o de conhecer como os enfermeiros constroem suas posturas pedagógicas para realização de atividades de Educação Continuada.

Verificou-se que todos os profissionais se disseram aptos para realizar Educação Continuada no local de trabalho, apesar de não terem tido formações e/ou capacitações pedagógicas específicas para tal. Alguns profissionais citaram que a graduação foi imprescindível no auxílio de desenvolvimento de habilidades executivas da Educação Continuada, no entanto, considera-se que teve uma contribuição pouco relevante, já que se baseou no incentivo, no oferecimento de dicas e informações.

Observou-se que em decorrência do ainda vigente modelo tradicional de ensino, alguns profissionais "repetem", na Educação Continuada, essa forma de apenas repasse de conhecimentos, tendo, portanto, uma postura pedagógica mais tradicional, construída na prática e por meio de uma busca pessoal.

Reforça-se a necessidade de construção de uma postura pedagógica mais dinâmica e inovadora, tendo como sugestão o uso das metodologias ativas como método de ensino, tornando a Educação Continuada mais atrativa e produzindo maior eficiência e eficácia das atividades educativas desenvolvidas.

Por fim, destaca-se a necessidade de desenvolvimento de novas pesquisas que abarquem o mesmo tema, porém, que tenha abrangência maior em outros campos de atuação da enfermagem, como a atenção básica ou atenção especializada, a fim de fomentar as discussões sobre práticas pedagógicas na saúde para formação contínua de trabalhadores em saúde. 


\section{Referências}

AMARO, M. de O. F.; MENDONÇA, E. T. de; CARVALHO, C. A. de; NAKADA, K. N.; SIMAN, A. G.; FERREIRA, N. da C. S. Concepções e práticas dos enfermeiros sobre educação permanente no ambiente hospitalar. Arquivos de. Ciências da Saúde UNIPAR, Umuarama, v. 22, n. 2, p. 87-94, 2018.

ARRUDA, L. P.; MOREIRA, A. C. A.; ARAGÃO A. E. A. Promoção da saúde: Atribuições do enfermeiro como educador na Estratégia Saúde da Família. Essentia, Sobral, v.16, n.1, p. 183-203, 2014.

BARDIN L. L'analyse de contenu, $1^{\text {a }}$ edição. Lisboa: Presses Universitária France, 1977.

BAPTISTA, G. L.; CUNHA, M. I. A dimensão educativa nos cursos de graduação em enfermagem: um estudo sobre a formação inicial. Revista Conhecimento Online v.1, n.1, p.221, 2009.

BRASIL. PORTARIA Nº 198/GM Em 13 de fevereiro de 2004. Institui a Política Nacional de Educação Permanente em Saúde como estratégia do Sistema Único de Saúde para a formação e o desenvolvimento de trabalhadores para o setor e dá outras providências, 2004.

FERREIRA JÚNIOR, M. A. F.; GRÍGOLI, M. A. G. G.; IVO, M. L. O início das atividades de ensino do professor enfermeiro. Revista Científica Internacional. a.3, n.12, p.45-64, 2010.

FLORES, G. E.; OLIVEIRA, D. L. L.; ZOCCHE, D. A. Educação Permanente no contexto hospitalar: a experiência que ressignifica o cuidado em enfermagem. Trabalho, Educação $e$ Saúde, v. 14 n. 2, p. 487-504, 2016.

GERHARDT, T. E.; SILVEIRA D. T. Métodos de pesquisa. Coordenado pela Universidade Aberta do Brasil - UAB/UFRGS e pelo Curso de Graduação Tecnológica - Planejamento e Gestão para o Desenvolvimento Rural da SEAD/UFRGS. - Porto Alegre: Editora da UFRGS, 2009.

GIL, Antônio Carlos. Como elaborar projetos de pesquisa. 4a ed. São Paulo: Atlas, 2010.

GUBERT, E.; PRADO, M. L. do. Desafios na prática pedagógica na educação profissional em enfermagem. Revista Eletrônica de Enfermagem [Internet]. v.13, n.2, p.285-95, 2011.

JESUS, M. C. P. De; FIGUEIREDO, M. A. G.; SANTOS, S M. R.; DO AMARAL, A. M. M.; ROCHA, L. O.; THIOLLEN, M. J. M. Educação permanente em enfermagem em um hospital universitário. Revista da Escola de Enfermagem da USP. v.45, n.5, p.1229-36, 2011.

RESOLUÇÃO CNE/CES No 3, DE 7 DE NOVEMBRO DE 2001. Institui Diretrizes Curriculares Nacionais do Curso de Graduação em Enfermagem 
SALUM, N. C.; PRADO, M. L. A. educação permanente no desenvolvimento de competências dos profissionais de enfermagem. Texto Contexto Enfermagem, Florianópolis, v.2, n.23, p. 3018, 2014.

SOUZA, L. P. De; LIMA, M. G. De. Educação continuada em unidade de terapia intensiva: revisão da literatura. Journal Health Biologic Science, v.3, n.1, p.39-45, 2015.

\section{Como citar este artigo (Formato ABNT):}

SILVA, Vinareis Gomes da; CÂNDIDO, Aldrina da S. C. A Formação do Enfermeiro para a Realização da Educação Continuada. Id on Line Rev.Mult. Psic., 2018, vol.12, n.40, p.847-858. ISSN: 19811179 .

Recebido: 23/05/2018

Aceito: 24/05/2018 